\title{
880.
}

\section{NOTE ON THE RELATION BETWEEN THE DISTANCE OF FIVE POINTS IN SPACE.}

[From the Messenger of Mathematics, vol. xvIII. (1889), pp. 100-102.]

IN Lagrange's paper "Solutions analytiques de quelques problèmes sur les pyramides triangulaires" (Berlin Memoirs, 1773; Euvres, t. III., see p. 677), there is contained a formula for the relation between the distances from each other of five points in space; viz. this is

$$
\begin{aligned}
4 \Delta^{2} f= & \alpha(a+f-g)^{2}+\alpha^{\prime}\left(a^{\prime}+f-g^{\prime}\right)^{2}+\alpha^{\prime \prime}\left(a^{\prime \prime}+f-g^{\prime \prime}\right)^{2} \\
& +2 \beta\left(a^{\prime}+f-g^{\prime}\right) \cdot\left(a^{\prime \prime}+f-g^{\prime \prime}\right)+2 \beta^{\prime}(a+f-g)\left(a^{\prime \prime}+f-g^{\prime \prime}\right) \\
& +2 \beta^{\prime \prime}(a+f-g)\left(a^{\prime}+f-g^{\prime}\right),
\end{aligned}
$$

or, in a slightly altered notation, say

$$
\Pi=-4 \Delta^{2} f+\left(\alpha_{1}, \alpha_{2}, \alpha_{3}, \beta_{1}, \beta_{2}, \beta_{3} \chi a_{1}+f-g_{1}, a_{2}+f-g_{2}, a_{3}+f-g_{3}\right)^{2}=0,
$$

where, if the points are called $1,2,3,4,5$, then

$$
\begin{array}{lcl}
c_{1}, c_{2}, c_{3} & \text { are the squared distances } & 23,31,12, \\
a_{1}, a_{2}, a_{3} & " & 41,42,43, \\
g_{1}, g_{2}, g_{3} & " & 51,52,53,
\end{array}
$$

and

$$
f \quad \text { is the squared distance } 4.5 \text {. }
$$

The values of $\alpha_{1}, \alpha_{2}, \alpha_{3}, \beta_{1}, \beta_{2}, \beta_{3}$, in terms of these squared distances, are

$$
\begin{aligned}
& \alpha_{1}=-\left(a_{2}-a_{3}\right)^{2}+2 c_{1}\left(a_{2}+a_{3}\right)-c_{1}{ }^{2}, \\
& \alpha_{2}=-\left(a_{3}-a_{1}\right)^{2}+2 c_{2}\left(a_{3}+a_{1}\right)-c_{2}{ }^{2}, \\
& \alpha_{3}=-\left(a_{1}-a_{2}\right)^{2}+2 c_{3}\left(a_{1}+a_{2}\right)-c_{3}{ }^{2}, \\
& \beta_{1}=-\left(a_{3}-a_{1}\right)\left(a_{1}-a_{2}\right)+2 c_{1} a_{1}-c_{2}\left(a_{1}+a_{2}\right)-c_{3}\left(a_{3}+a_{1}\right)+c_{2} c_{3}, \\
& \beta_{2}=-\left(a_{1}-a_{2}\right)\left(a_{2}-a_{3}\right)+2 c_{2} a_{2}-c_{3}\left(a_{2}+a_{3}\right)-c_{1}\left(a_{1}+a_{2}\right)+c_{3} c_{1}, \\
& \beta_{3}=-\left(a_{2}-a_{3}\right)\left(a_{3}-a_{1}\right)+2 c_{3} a_{3}-c_{1}\left(a_{3}+a_{1}\right)-c_{2}\left(a_{2}+a_{3}\right)+c_{1} c_{2} .
\end{aligned}
$$


After some reductions, the value of $4 \Delta^{2}$, in terms of these squared distances, is found to be

$$
\begin{aligned}
4 \Delta^{2}= & c_{1}\left(a_{3}-a_{1}\right)\left(a_{1}-a_{2}\right)+a_{1}\left(-c_{1}^{2}+c_{1} c_{2}+c_{1} c_{3}\right)-c_{1} c_{2} c_{3} \\
+ & c_{2}\left(a_{1}-a_{2}\right)\left(a_{2}-a_{3}\right)+a_{2}\left(-c_{2}{ }^{2}+c_{2} c_{3}+c_{2} c_{1}\right) \\
+ & c_{3}\left(a_{2}-a_{3}\right)\left(a_{3}-a_{1}\right)+a_{3}\left(-c_{3}{ }^{2}+c_{3} c_{1}+c_{3} c_{2}\right)
\end{aligned}
$$

which is, in fact,

$$
8 \Delta^{2}=\left|\begin{array}{ccccc}
. & 1, & 1, & 1, & 1 \\
1, & . & c_{3}, & c_{2}, & a_{1} \\
1, & c_{3}, & . & c_{1}, & a_{2} \\
1, & c_{2}, & c_{1}, & . & a_{3} \\
1, & a_{1}, & a_{2}, & a_{3}, & .
\end{array}\right|
$$

where observe that each term of the determinant contains the factor 2 .

By the formula in my paper "On a theorem in the Geometry of Position," Camb. Math. Jour., t. II. (1841), pp. 267-271, [1], (introducing into it the present ,notation), the relation between the distances of the five points is given in the form

$$
\Omega=\left|\begin{array}{cccccc}
. & 1, & 1, & 1, & 1, & 1 \\
1, & . & c_{3}, & c_{2}, & a_{1}, & g_{1} \\
1, & c_{3}, & . & c_{1}, & a_{2}, & g_{2} \\
1, & c_{2}, & c_{1}, & . & a_{3}, & g_{3} \\
1, & a_{1}, & a_{2}, & a_{3}, & . & f \\
1, & g_{1}, & g_{2}, & g_{3}, & f, & .
\end{array}\right|=0 .
$$

The equations $\Pi=0, \Omega=0$ should therefore agree with each other; we have in $\Omega$ the term

which is

$$
-f^{2}\left|\begin{array}{cccc}
1, & c_{3}, & . & c_{1} \\
1, & c_{2}, & c_{1}, & . \\
\cdot & 1, & 1, & 1 \\
1, & . & c_{3}, & c_{2}
\end{array}\right|
$$

$$
=-f^{2}\left(c_{1}^{2}+c_{2}^{2}+c_{3}^{2}-2 c_{2} c_{3}-2 c_{3} c_{1}-2 c_{1} c_{2}\right)
$$

and similarly in $\Pi$ we have the term

$$
f^{2}\left(\alpha_{1}+\alpha_{2}+\alpha_{3}+2 \beta_{1}+2 \beta_{2}+2 \beta_{3}\right)
$$

which is easily shown to be

$$
=-f^{2}\left(c_{1}^{2}+c_{2}^{2}+c_{3}^{2}-2 c_{2} c_{3}-2 c_{3} c_{1}-2 c_{1} c_{2}\right) ;
$$

and it thus appears that we have identically $\Pi=\Omega$. 
It is to be remarked that, in Lagrange's form, the points 4 and 5 are regarded as determined each of them by means of its squared distances from the vertices of the triangle 123, and that the formula gives (by a quadratic equation) the squared distance 45 ; but that nevertheless the two points 4 and 5 do not present themselves symmetrically in the formula; in fact, $\Delta^{2}$ and the coefficients $\left(\alpha_{1}, \alpha_{2}, \alpha_{3}, \beta_{1}, \beta_{2}, \beta_{3}\right)$ of the formula relate all of them to the tetrahedron 4123; as noticed in the paper, $\Delta=6 \times$ volume of tetrahedron; $\sqrt{ }\left(\alpha_{1}\right), \sqrt{ }\left(\alpha_{2}\right), \sqrt{ }\left(\alpha_{3}\right)$ are the doubled areas of the faces $423,431,412$ respectively, and the doubled area of the face 123 is

$$
=\sqrt{ }\left(\alpha_{1}+\alpha_{2}+\alpha_{3}+2 \beta_{1}+2 \beta_{2}+2 \beta_{3}\right) ;
$$

it may be added that

$$
\beta_{1} \div \sqrt{ }\left(\alpha_{2} \alpha_{3}\right), \quad \beta_{2} \div \sqrt{ }\left(\alpha_{3} \alpha_{1}\right), \quad \beta_{3} \div \sqrt{ }\left(\alpha_{1} \alpha_{2}\right),
$$

are equal to the cosines of the dihedral angles at the edges $41,42,43$ respectively. 